\title{
Comparability Between Simulation and Reality in Apparel: A Practical Project Approach from 3D-Body Scan to Individual Avatars and from 3D-Simulation in Vidya to Fitted Garments
}

\author{
Michael ERNST ${ }^{\star a}$, Anke RISSIEK* ${ }^{\text {b }}$ \\ ${ }^{\mathrm{a}}$ Niederrhein University of Applied Sciences, Germany; \\ ${ }^{\mathrm{b}}$ Human Solutions, Germany
}

\begin{abstract}
Nowadays the product development process in the clothing industry- and especially the technical product development process- becomes faster and faster.

Therefore the availability of innovative 3D Body scanning technology and 3D CAD tools enables to break new grounds and improve the process design entirely in a sustainable manner [1].

This paper presents a practical approach on the way to a digital product development process in the apparel industry and points out the status quo of tools used in this field.
\end{abstract}

Keywords: 3d body scan, avatar, 3D simulation, product development, fit control

\section{Introduction}

Today the complete textile supply chain is confronted with an ever-increasing number of collections to markets in ever-decreasing time frames. Additionally the requirements to product development augment enormously based to the need of global sales and marketing. Fashion companies produce their products for global markets which are characterized by critical customers and their diminishing willingness to spend much money for clothes that fit only fairly good. Beside the price fit is the most important buying factor.[11]

Because of the ever increasing time pressure in product development processes and collection development the fashion industry is faced to paradigm changes that have already been finished 20 years ago in the automotive sector: (1) In the future the product development will be realized no longer in 2D but in 3D and (2) the industry will get used to make decisions for their collections based on virtual product development and -visualization apart from real samples.

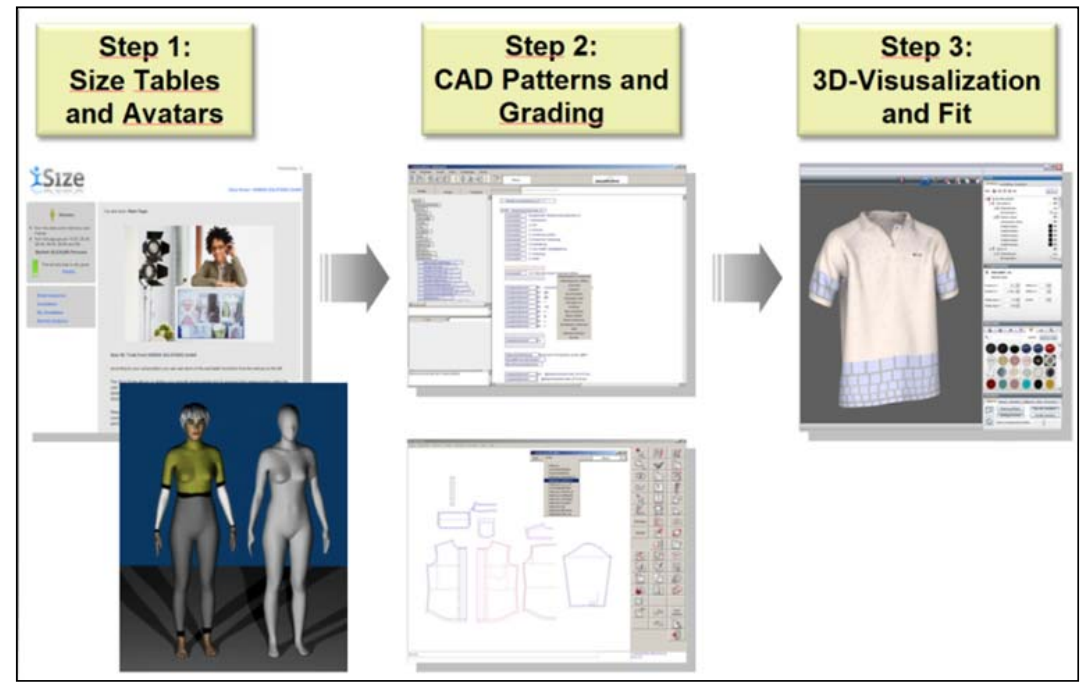

Fig.1: Future process within the process chain for virtual product development and fit optimization

michael.ernst@hs-niederrhein.de ; +49 2161 186-6080; www.hs-niederrhein.de anke.rissiek@human-solutions.com; +49 631 303-5645; www.human-solutions.com 
3D pattern design and virtualized product development processes provide answers for rethinking processes and gradually carrying more steps out digitally related by saves of time and money. 3D CAD systems can reduce frustrating and cost-intensive iterations between design, pattern making, sample production and try-on sessions with fit models or on physical manikins.[7]

The most important global CAD suppliers (Gerber (USA), Lectra (France), Optitex (Israel) and assyst (Germany) already offer first solutions for the 3D simulation of pattern.[8] However they are nowadays more or less used to visualize the results of different development steps and not for visualizing the fit of the garment. By importing avatars into a pattern making software which has 3D virtual try-on feature, it is possible to rotate 3D images to see how the garment would look on a person. Depending on the fit and style preferences, the garment pattern could be also altered by the users themselves if they have access to the software or if an interface can be created to modify patterns.

The advantages of the 3D simulation and visualization tools are:

- $\quad$ high quality and efficiency in the development process

- communication basis for cross-border and cross-site coordination

- integration of real body shapes and volumes in design and pattern development

- reduced need for physical prototypes

The comparability between 3D simulation and reality in apparel is one of the main challenging tasks for industry: Only if the 3D simulation brings out the same results as real fitting tests on real models the new technology will find acceptance in the pattern development departments in the future.

\section{Research methodology}

In this paper only a small part of the project results is presented: the complete one includes investigations on basic block construction generally- skirts, trousers, dresses, jackets, influences of material characteristics, product development of dresses using scanatars and industry trials comparing e.g. men's shirts, jackets and trousers simulation to origin house models and the adaptation to rapid prototyping.

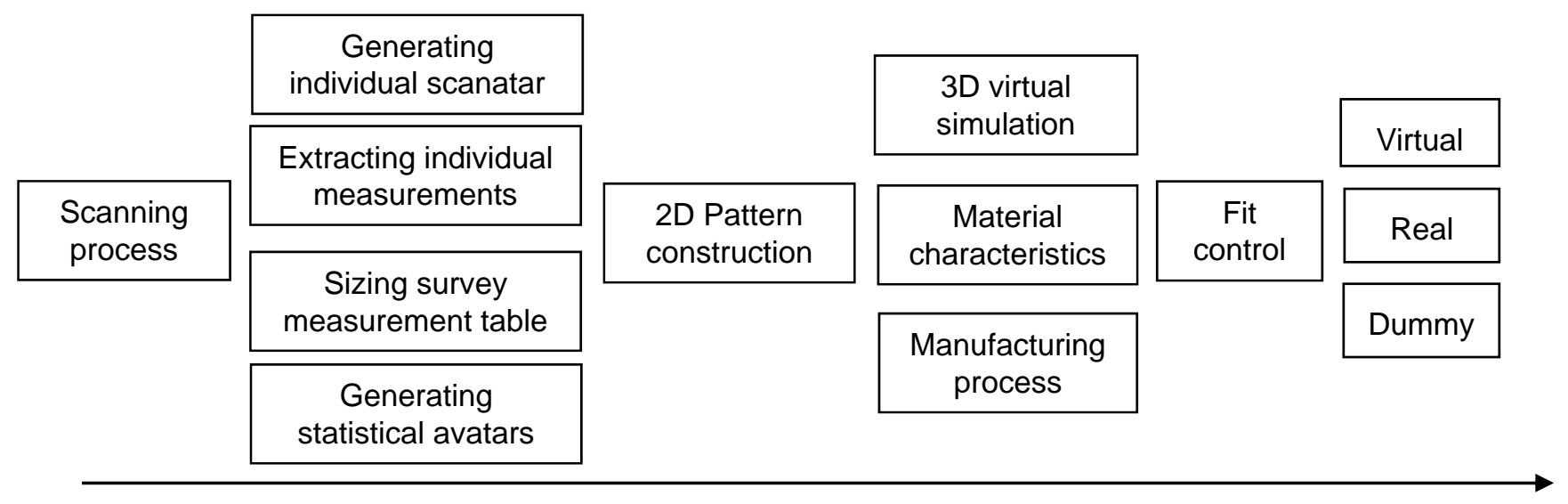

Fig. 2: Research methodology for a virtual product development process design

In a first step individual scantars are generated based on an innovative scanning process and imported to a 3D CAD program. In a second step pattern constructions for basic skirt blocks were investigated and adapted to individual measurements obtained from the individual 3D scan. The skirts are simulated on scantars in a 3D CAD program and compared to realistic manufactured individual skirts. 


\section{From 3D bodyscan to 3D avatars}

3D body scanning technologies have nowadays achieved significant advancement and acceptance in the area of sizing surveys, size prediction and mass customization. 3D body scanners can be used to create realistic images of a person and achieve exact body measurements of individuals. Body scan data increase the number and accuracy of measurements used in pattern construction and size prediction. The combination of virtual try-on with size prediction will not only provide consumers with the brands and sizes that fit their measurements and proportions best, but will also let them virtually view garments on their scan and choose the design they like best. This process combines objective fit information with fit preference - the most realistic way of satisfying customers.

3D body scans have specific characteristics which are influenced mainly by the measurement principle (laser, white light etc.) itself, the scanner construction and by the measurement position of the scanned subject. No matter which scanner is used, the raw data show gaps in the point cloud, especially under the arms and in the crotch area, that result from shadowing effects.

\subsection{General Requirements on avatars}

The body scan (in dependence from the individual measurement posture) has to provide exact body measurements with few gaps in the point cloud for pattern adaption and generation what indicates a special scanning posture. Actual analysis of the nowadays simulation tools have shown that there are high requirements on the exactness and topology of the avatar. Only if the avatars are correct, the pattern will fit to the individual person, in reality and in virtual try-on processes. That would lead to a classic scanning posture which does not optimally represent a relaxed posture.

On the one hand the scanning posture should be as similar as possible to a normal posture of an individual in a relaxed standing position. Only if the avatar posture is comparable with the relaxed posture of the scanned subject the fitting results in the virtual and in the real world will be comparable, too, and therefore lead to realistic fit assessments.

Fitting tests in 3D simulation tools exceedingly depend on the exactness of the used avatars whereupon exactness means in this case (1) correspondence with the body measurements of the size table that is used for pattern construction, and at the same time (2) correspondence in 3D body form of the real target customer that at the end will wear the garment and judge fit.

Human Solutions decided to resolve this apparent contradiction by producing 2 scans, a relaxed and a scan in classic measurement position for avatar generation. The two scans are made just one after the other and loaded on one screen.

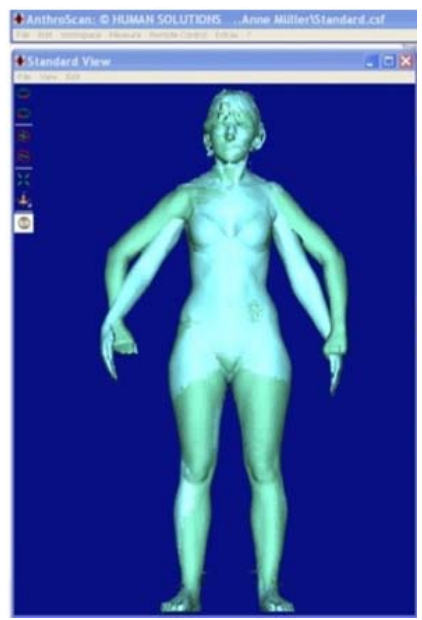

Fig. 3. Two scans (loaded into each other) as a basis for avatar generation

\subsection{Different approaches to avatars}

Nowadays avatars can be developed according to three different basic approaches:

Generic avatars: Most simulation engines work with artificial human bodies, so called generic avatars, that fulfill more aesthetic aspects than requirements related to correct body proportions, dimensions and $3 \mathrm{D}$ shape. They fulfill perfectly the requirements of the designers because they are 
available in different defined looks with perfect hair, makeup, manicured hands and feet. The generic avatars consist of a parametric model that can easily be adapted to different body measurements. Creating an avatar far away from standard body measurements often causes artefacts in body shapes that are very obvious and that lead to serious problems in simulation.

Individual avatars (Scanatars): Scanatars consist of avatars from individual persons that have been scanned with a body scanner. As they are identical representations of the individual person they are identical in body measurements and shapes. Normally the body scanners do not generate color information so that hair and makeup is not separable from the body geometry. The process of generation of scanatars is described in detail in chapter. 3.3.

Statistical avatars: Real statistic representatives of standard sizes have been up to now standard fashion manikins, which should represent the three-dimensional body in relation to the characteristic sizes stored in standard size tables. These sizes can be measured at the relevant points on the standard fashion manikin. However, a reference regarding body shape or posture is not contained in the table of sizes and will therefore not be converted for the standard fashion manikins. But precisely these body shape and posture parameters have such a decisive influence on the size \& fit of an item of clothing.[10]

Human Solutions has developed a new technology to create statistical avatars directly from scan data. All the 3D scans - which primary body measurements are covered by a company specific or a standard size table - are selected, using the primary dimensions and tolerance areas of the clothing table.

Every human body is created symmetrically, but in a very limited way. Differing shoulder angles or oblique pelvis positions have a considerable impact on the sizing and fitting of clothing. The basic approach in software application is to mirror the person completely and the average it with the unsymmetrical original. Normally the averaged avatar does not exactly fit to the target body measurements of a size table because of the enormous output variability of all the individual scans. Therefore different parametric morphological operations have been developed that work with synthetic rescaling operators for the length and for the width dimensions to adapt the avatar to the predefined measurements.[6]

Within the SizeGERMANY measurement survey statistical avatars that can be used in vidya as representatives of the average body forms for standard sizes have been part of the project results. The consistency of the measurements and form concept for real manikins and virtual fitting guarantees the comparability of the fit (measurements according to the size table, identical sizes, identical body shapes, corresponding measuring lines on bust with sizes on the avatar).[9] Scanatars and statistical avatars can take up any pose in vidya 20.11. Users can animate a desired model as and when required - including movements like taking a step, stretching out an arm and sitting down.

\subsection{Innovative methodology of generating individual avatars (scanatars) in the project}

Within the project the students have been individually scanned with the body scanner VITUS XXL from Human Solutions and scanatars for vidya have been generated. The example scan and avatar seen below is also used in the real and virtual try-on chapters.

In a first step the critical geometry is completed with synthetical geometry using NURBS, a mathematical model commonly used in computer graphics for generating and representing curves and surfaces which offers great flexibility and precision for handling both analytic and freeform shapes. The completed surfaces are than used for a reverse engineering (Poisson method) that leads to a complete surface structure without creating any gaps ("webs") under the arm or between the legs.

The generating process for individual avatars consists of three process steps which are (1) check surface, (2) generate surface and check landmarks and (3) save as vidya avatar. Human Solutions has built up a software wizard that conducts the user through the complete process and therefore guaranties a successful avatar generation. 


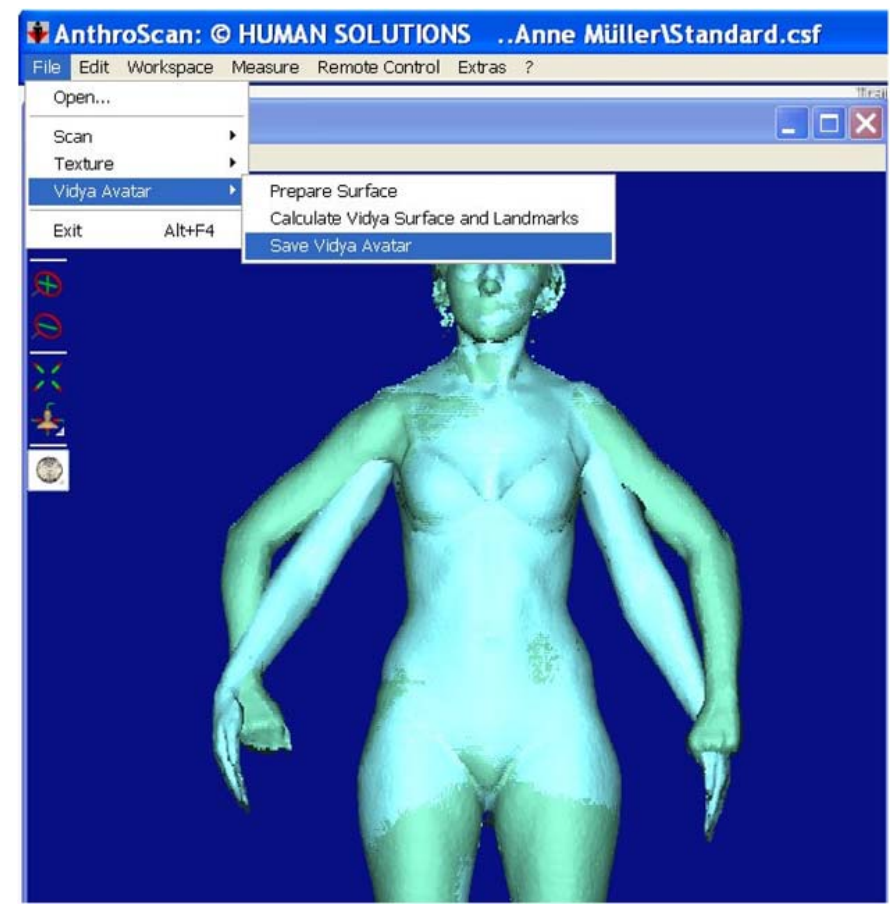

Fig. 4: Avatar generation tool

In the first process step the two scanning postures - standard and relaxed - are checked for completeness of the surface and for appropriateness for the avatar generation process. Relaxing scanning positions with loose-hanging arms close to the body are critical for the generation of a closed surface geometry, are therefore checked and identified artefacts can be corrected by processing the basic geometry.

In the second step the critical areas under the arms and in the crotch are checked and the user can interactively correct the proposed posture and the depth of the slits by editing the start and the end points. The shown polygon defines the amount of slit modeled in a consecutive step after the wizard is finished.

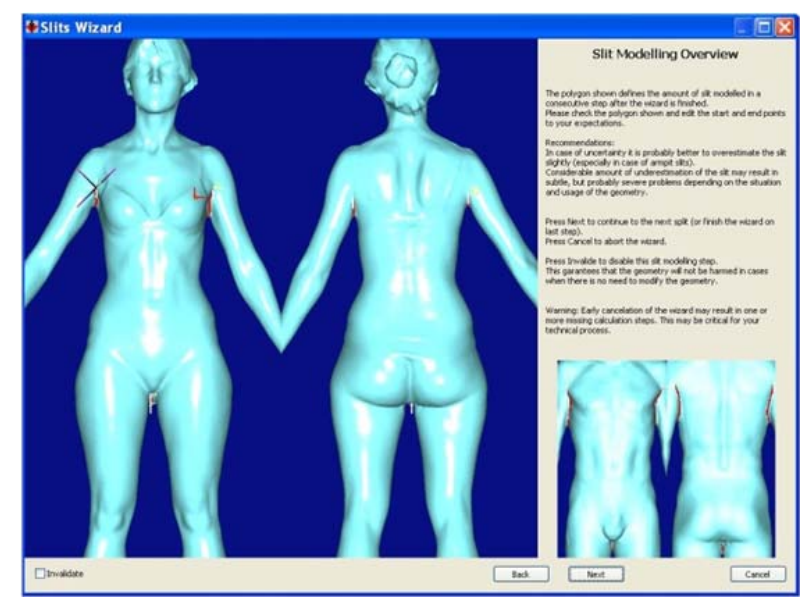

Fig. 5: Slits wizard for interactive correction of the slits in the armpit and in the crotch area

In the third step the vidya avatar is generated and the landmarks that are important for a quick and consistent pre-positioning in vidya are visualized and can be interactively corrected as well as the body measurements generated from the scan. 


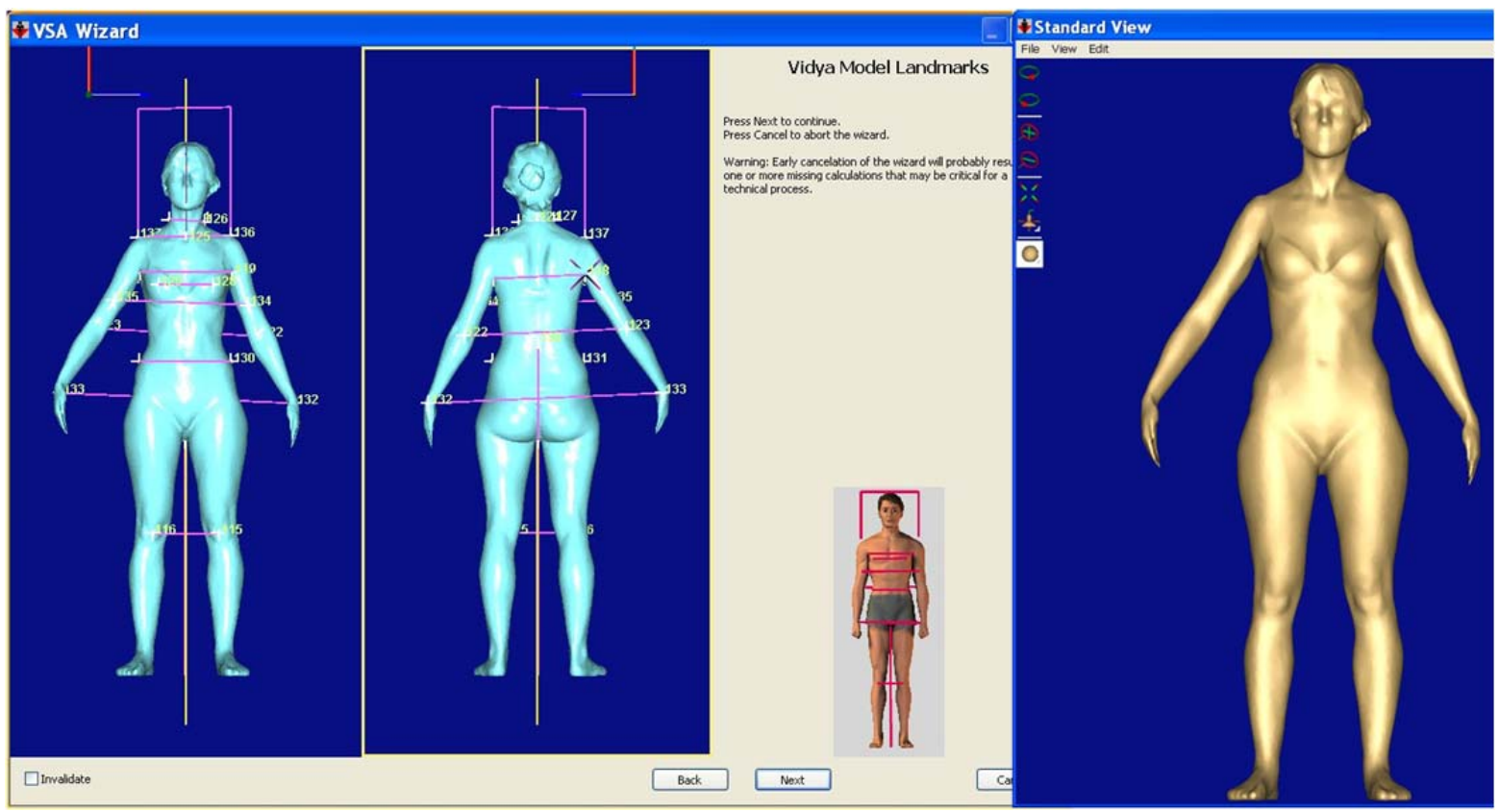

Fig. 6: Avatar with vidya model landmarks prepared for prepositioning

\section{Pattern construction}

For the first practical approach the virtual product development process from individual body scans to fitted garments was investigated by choosing the product of a simple basic skirt.

Therefore the methodology was split in two sections:

1. Investigations on fit control of different basic block constructions by using SizeGERMANY measurements, a SizeGERMANY avatar (statistical avatar) and different materials.

2. Investigations on fit control by using individual measurements extracted from body scans and so called scanatars.

\subsection{Basic block skirt- SizeGERMANY}

Pattern construction was done using cadassyst- one of the most powerful 2D CAD program used in the apparel industry $[3,7]$.

Two different pattern construction systems have been investigated in a first approach:

- Contec Optikon (University of Applied Sciences Niederrhein)

- Mueller\&Son (Mueller\&Son; Duesseldorf)

The basic skirt constructions differ in ease for hip and waist, the waist to hip measurement calculation and the distribution of hip to waist ratio to front and back dart and to the side seam hip curve.

Furthermore the Contec system splits into two sizing groups- the small one (Size 34-46) and the large one (Size 46-56) with Size 46 defined as border size.

The measurements for the constructions are taken from the latest sizing survey carried out in Germany in 2009- available on the I-Size portal- the portal for international sizing systems.

As basic size German N 38 was chosen in the small sizing group and grading was done by using a conventional step method.

In Figure 7 the graded basic block including the straight waistband from Contec system is shown in cadassyst. There is no seam allowance attached and the darts are not headed yet. 


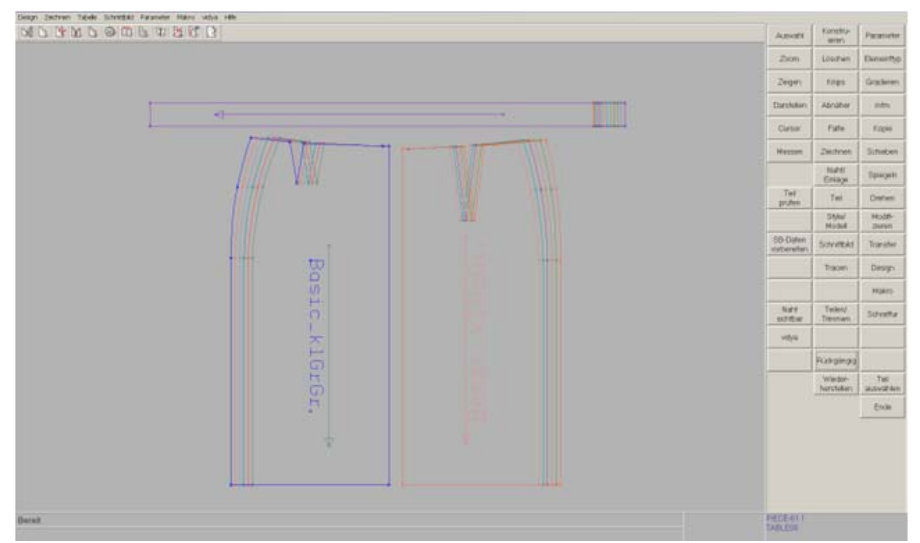

Fig. 7: Basic block in cadassyst with straight waistband, graded for the small sizing group 34-44

\subsection{Individual pattern/mass customized skirts}

The measurements for the individual pattern construction are detected by body scanning of 10 female subjects using the latest technology of ANTHROSCAN from Human Solutions.

Depending on individual body shapes and measurements the localization of the landmarks waist band and hip girth were modified for fit improvement. The distance waist to hip was calculated by subtracting the hip height from the waistband front height and the skirt length was chosen individually. To break down the number of parameters the waistband was given with $4 \mathrm{~cm}$ width compulsory- also a slit in center back for mobility. The localization and direction of the darts of the front and back panel are given by the basic block construction and are not modified for comparability. The seam allowance for the side seams and center back is given with $1 \mathrm{~cm}$. To avoid an influence of different hem stitching methods on the real product the hem seam allowance was given with $4 \mathrm{~cm}$ and generally blind-stitched.

\section{Simulation technology}

\subsection{Preparation of pattern}

The pattern created by using the 2D CAD program cadassyst are prepared in the so called vidyacad program- which is a special version of cadassyst and enables the customer to run through a user-friendly process where [4]:

- Pattern for simulation are selected

- Pattern properties are defined (material, interlining, symmetry, grain line, layer, positioning point..)

- Virtual sewing is done (seam allowance, ease distribution, pleated seam..)

- Sewing lines are defined (hem, slit, top stitching..)

- Applications are positioned

- Pleats are defined (cad pleats by notches, lapel fold line..)

The skirt pattern are in a prototyping state and not a production state which simplifies the preparation. The pattern are stored in a 3D style file containing all the information necessary for the simulation process and sent directly to vidya- the 3D simulation program. This link enables the user to exchange information between both programs- e.g. sending information for positioning back to 2D, make changes and actualize the simulation quickly.

\subsection{Material characteristics}

The materials used in this research have been investigated on their characteristics by assyst (Munich) using a special testing kit and scanned for design and color and imported to vidya system. The following material properties for warp weft and bias direction are necessary to know for simulation and the data have been imported to vidya: 
Table 1. Material characteristics

\begin{tabular}{|l|l|l|l|l|l|}
\hline & & $\begin{array}{l}\text { M1 grey } \\
\text { wool }\end{array}$ & $\begin{array}{l}\text { M2 } \\
\text { Knit jersey }\end{array}$ & $\begin{array}{l}\text { M3 pink } \\
\text { taffeta }\end{array}$ & $\begin{array}{l}\text { M4 } \\
\text { velveteen }\end{array}$ \\
\hline${\text { mass }\left[\mathrm{kgm}^{-2}\right]}^{\text {Attenuation }\left[\mathrm{s}^{-1}\right]}$ & & 0,21 & 0,25 & 0,23 & 0,22 \\
\hline Thickness [mm] & & 20 & 5 & 80 & 20 \\
\hline Coefficient friction & & 0,56 & 0,73 & 0,5 & 0,5 \\
\hline Elasticity [\%] & & 0,5 & 0,5 & 0,4 & 0,5 \\
& Warp & 102 & 103,98 & 100,5 & 101,14 \\
& Weft & 105 & 104,68 & 100,5 & 101 \\
& Bias & 120 & 115,3 & 103,5 & 102,1 \\
\hline Compressibility [\%] & Warp & 100 & 74,1 & 100 & 100 \\
& Weft & 100 & 69,5 & 100 & 100 \\
\hline E-module & Warp & 1,67 & 18,45 & 22,5 & 1 \\
(tension/bias)[Pam $\left.{ }^{-1}\right]$ & Weft & 0,67 & 19,5 & 26,5 & 1 \\
& Bias & 0,17 & 0,06 & 13 & 0,5 \\
\hline Flexural rigidity & Warp & 0,64 & 0,63 & 0 & 0,52 \\
& Weft & 0,51 & 0,51 & 0 & 0,47 \\
\hline E-module (bending) $\left[\right.$ Pam $\left.^{-1}\right]$ & Warp & 0,2 & 0,18 & 0,47 & 0,12 \\
& Weft & 0,16 & 0,13 & 0,89 & 0,11 \\
\hline
\end{tabular}

\subsection{Real Time Simulation in Vidya}

Vidya is a powerful 3D simulation program for the apparel industry and offers a real time simulation which can be started and stopped all the time.

The prepared pattern sent from vidyacad is now fitted with a theme where all the details: material design, material characteristics, buttons and buttonholes, fold lines, stitching, attachments are fixed. Also the avatar or scanatar, the environnement, the simulation accuracy/grid size are selected now. During the active simulation virtual pins can be used to pull the fabric, material design and parameters can be changed quickly by drag and drop, pattern pieces can be hidden, body distance control, pressure and tension map can be shown [5].

\subsection{Manufacturing process}

Pattern files created by cadassyst are layed with layassyst to achieve so called cutfiles. These cutfiles are sent to an automatically running cutting system (One layer, Zündt) with conveyer system for cutting- thus saving time and achieving accuracy.

The waistband was interlined using Freudenberg Vilene waistband interlining with fixed $4 \mathrm{~cm}$ width, bonded on with a continuously running bonding machine (Meyer).

For sewing industrial sewing machines were used:

- Dürkopp 271 single needle plain stitching machine 301 , stitch length $2,5 \mathrm{~mm}$

- Rimoldi three thread overlock 504

- $\quad$ Strobel blind stitching machine 103

- Sewing thread: Amann saba 120

- Needle: SRG 70 (Groz-Beckert)

- Finishing was done by using industrial ironing equipment (Veit)

\section{Results}

The results presented in this paper include the time-lapse procedure of building up scanatars from 3D body scans and import them to a 3D CAD program, a virtual fit of basic block skirts and individual skirts compared to reality.

\subsection{Basic block investigation- SizeGERMANY data}

In the following figures, simulation results of the basic skirt obtained with vidya 3D simulation software are shown. The pattern was prepared in vidyacad with seams in center front and center back, one dart on front panel and one dart on back panel. 
The basic skirt based on the Contec pattern construction system shows a well fitted garment on the SizeGERMANY 38 avatar using material $\mathrm{M}_{1}$.as material of choice with typical body distance values for a tight fit. The results mainly depend on the chosen material and can differ in a broad range.
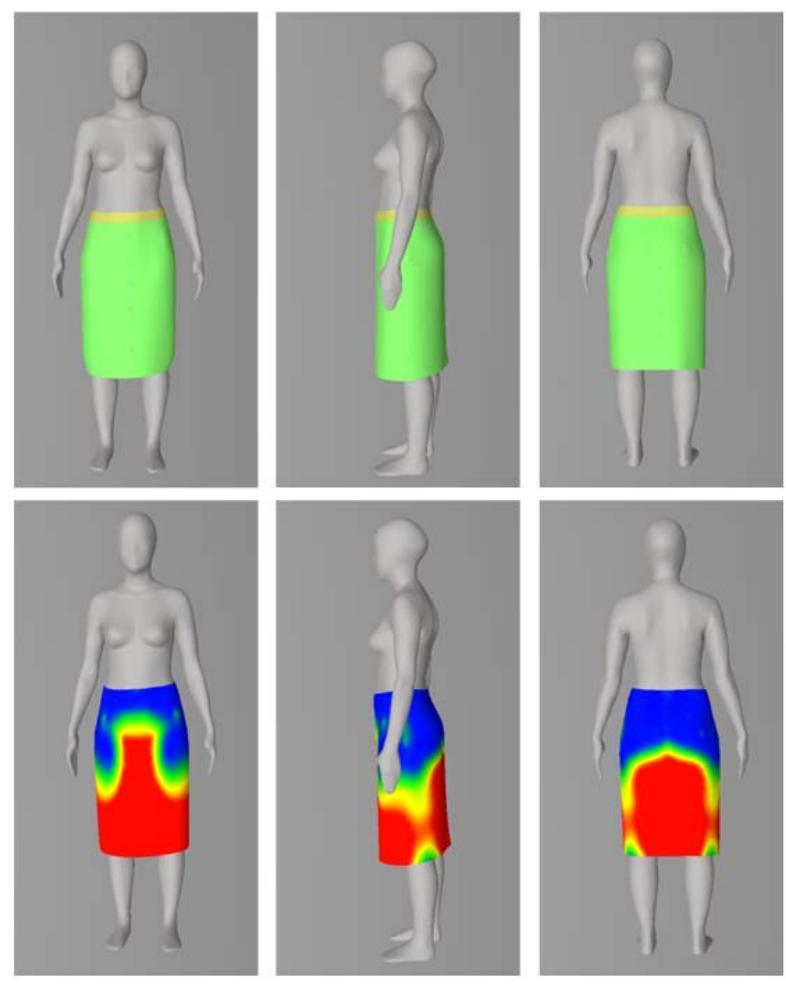

Fig. 8: Basic block skirt Contec- fit control and body distance control on SizeGERMANY avatar 38. Body distance color mapping: blue minimum $0 \mathrm{~mm}$; red maximum $30 \mathrm{~mm}$; material $\mathrm{M}_{1}$

Simulation details and power of vidya Simulation is shown in Fig.9 - a front dart was virtually sewn in straight line and (as in reality it should be) in curved line to achieve best fit with material $\mathrm{M}_{1}$.. Also the simulation results show the smoother shape of the dart- fit to body and therefore the comparability to reality.

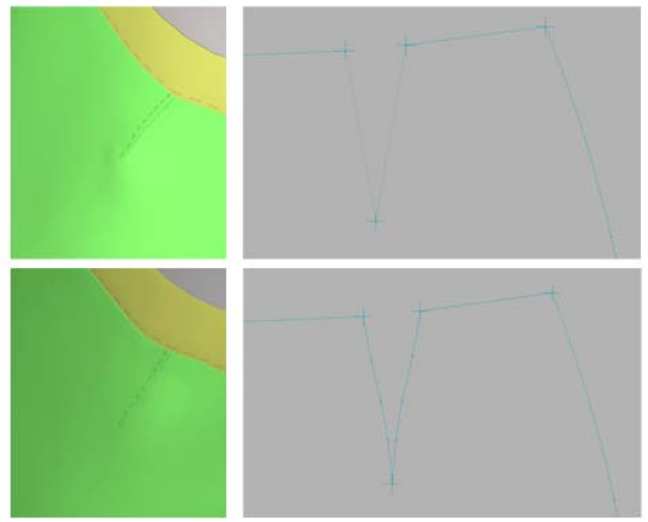

Fig. 9: Typical results of sewn darts- straight and curved in Cad-boiled and smooth in simuation

Figure 10 shows the influence of choosing different material characteristics $M_{1}-M_{4}$ for the simulation of the basic block skirt: The position of the skirt, the balance, the smoothness of hip curves and darts differ more or less depending on parameters. Draped pleats occur with the soft taffeta and velvet. 


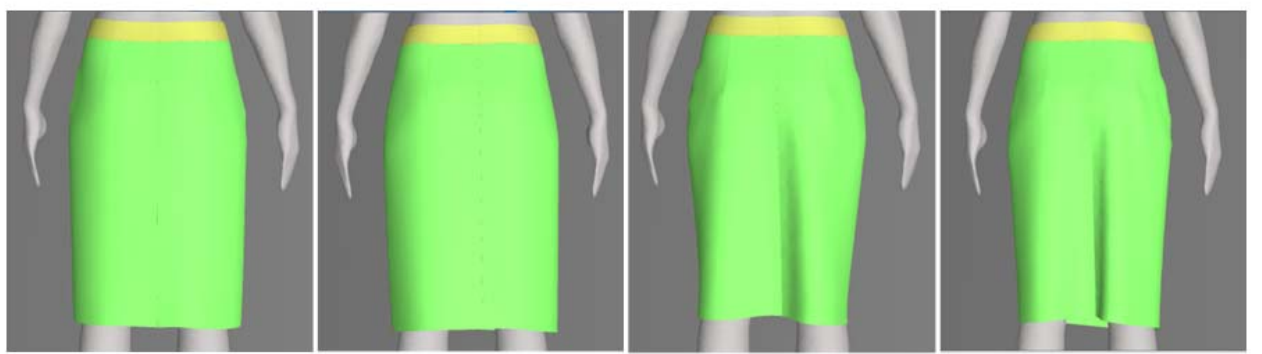

Fig 10: Typical results of basic block Contec, on SizeGERMANY Avatar 38, different materials $M_{1-4}$

\subsection{Basic block investigation- individual fit}

The following 3 examples show a small selection of the results obtained from the investigation on individual pattern construction and fit control done virtually by using data and scanatars from 3D body scans.

\section{Example 1}

Table 2. Measurements from individual scan for skirt basic block construction

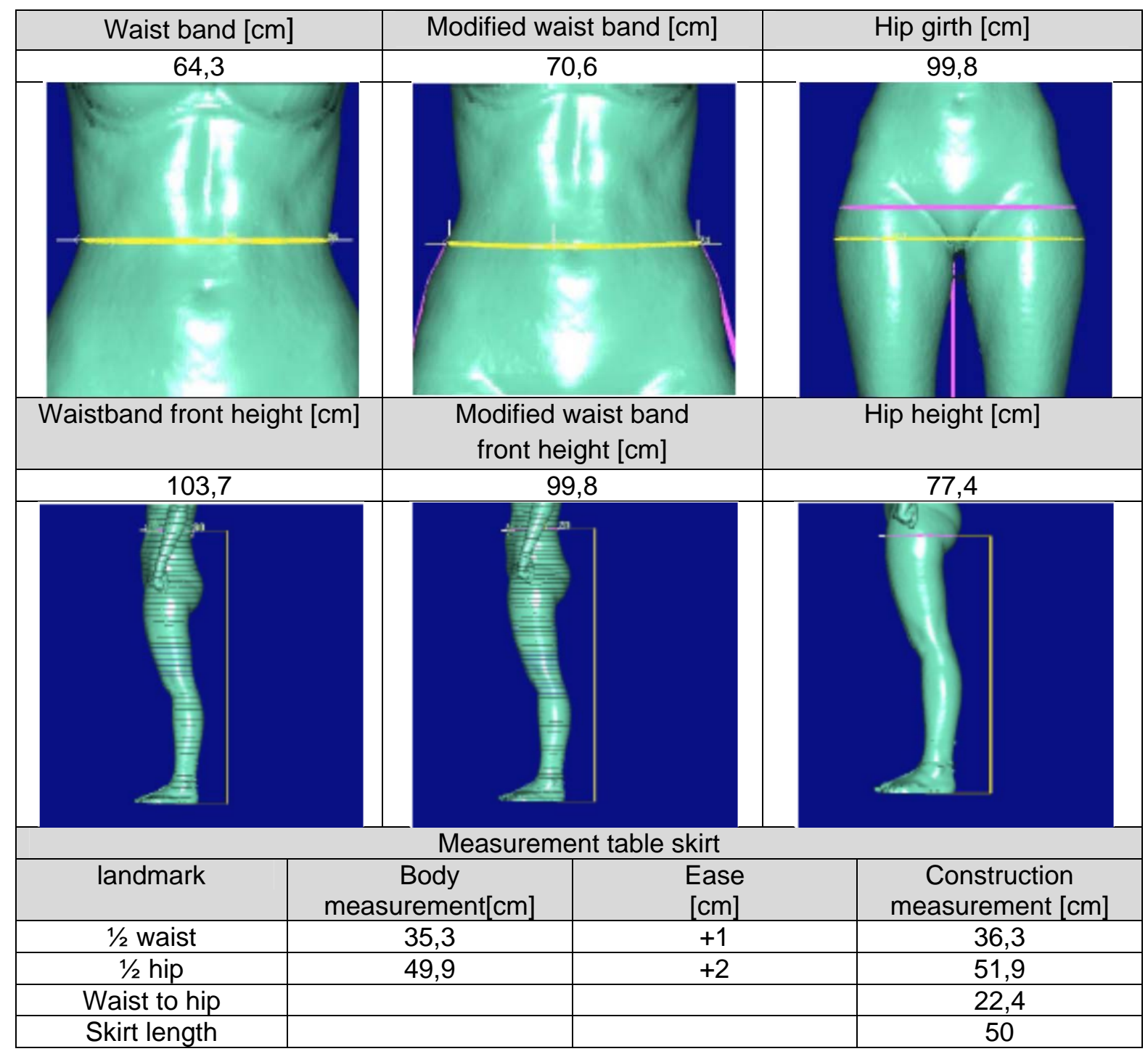



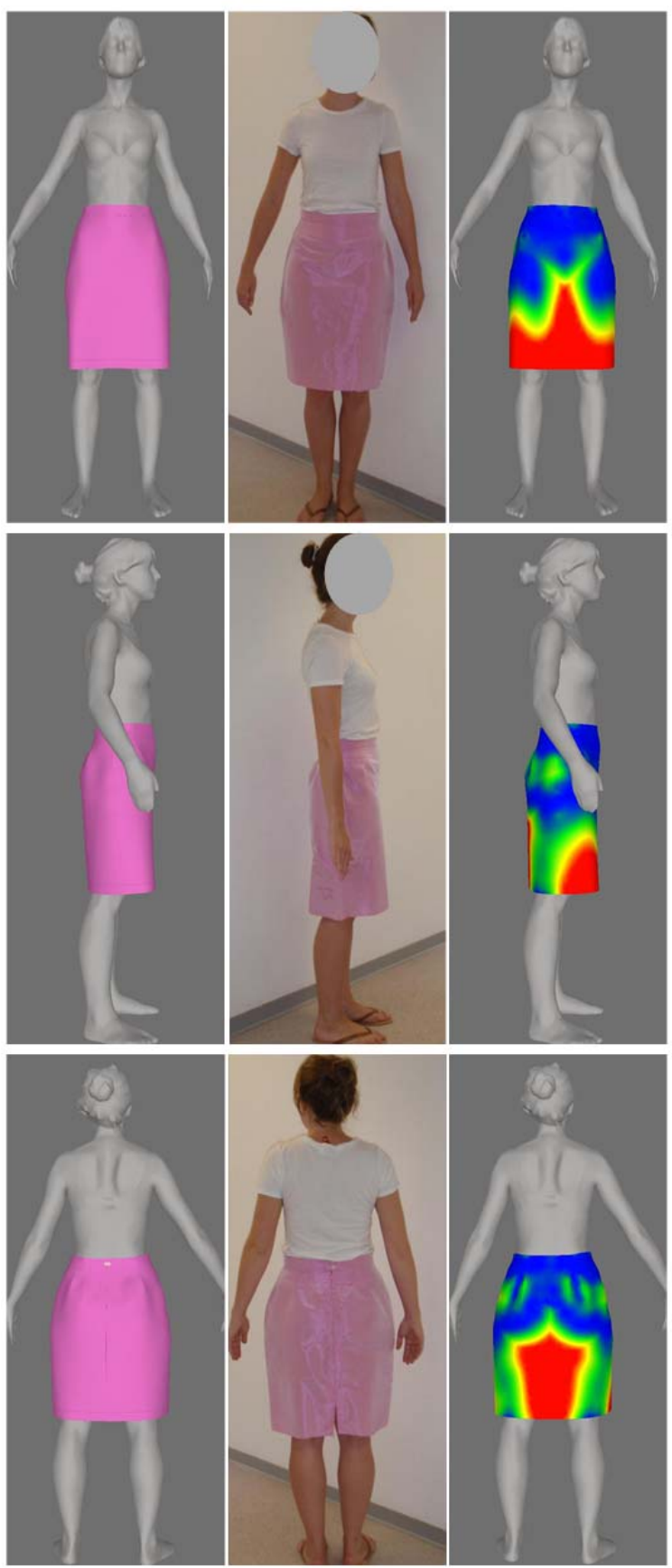

Fig.11: Results of virtual fitting on scanatar versus real fitting body distance colour mapping: blue minimum $0 \mathrm{~mm}$; red maximum $30 \mathrm{~mm}$; material $\mathrm{M}_{3}$

Example 1 shows the result of a successfully operating process design. The waist landmark has been changed (lowered) according to individual body shape in waist position and hence the calculation of the waist to hip measurement was adapted.

The ease for the basic construction was chosen as recommended by Contec for the small size group (table 2)- a second back dart was first chosen because of the individual body shape and detected as not necessary in the 3D fit control by using color mapping of body distance (Fig. 11). The darts are sewn in curved shape both in simulation and production. Only the simulation of the material itself with a little crinkle effect in reality is not perfect and in need of improvement. 


\section{Example 2}

Table 3. Measurements from individual scan for skirt basic block construction

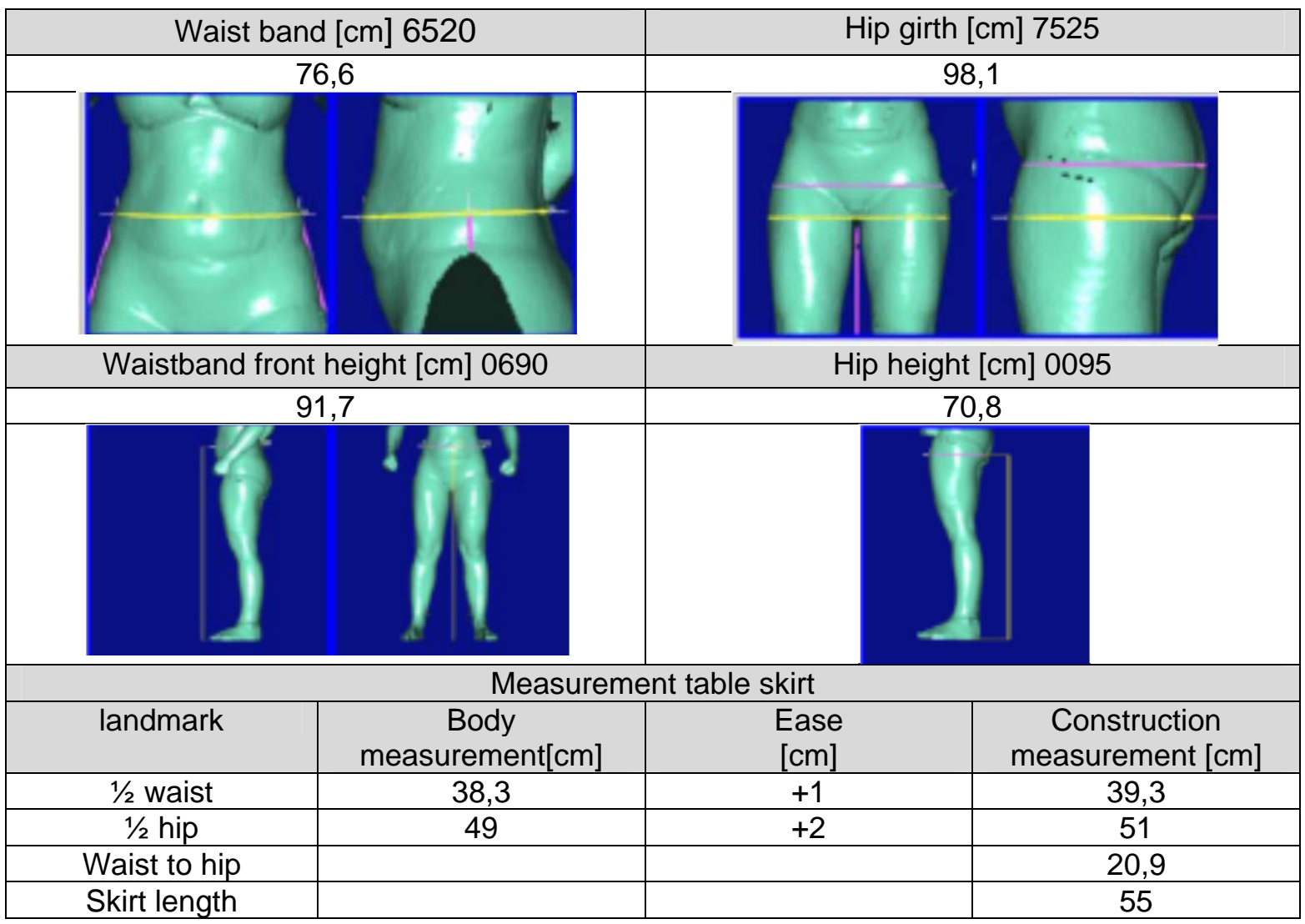

For example 2 the position of the landmarks waist band and hip girth could be used directly from the body scan without change. As shown in Fig. 12 the results and therefore the prediction is good and therefore useful to detect the critical parts: center front and balance of the skirt on side view. 

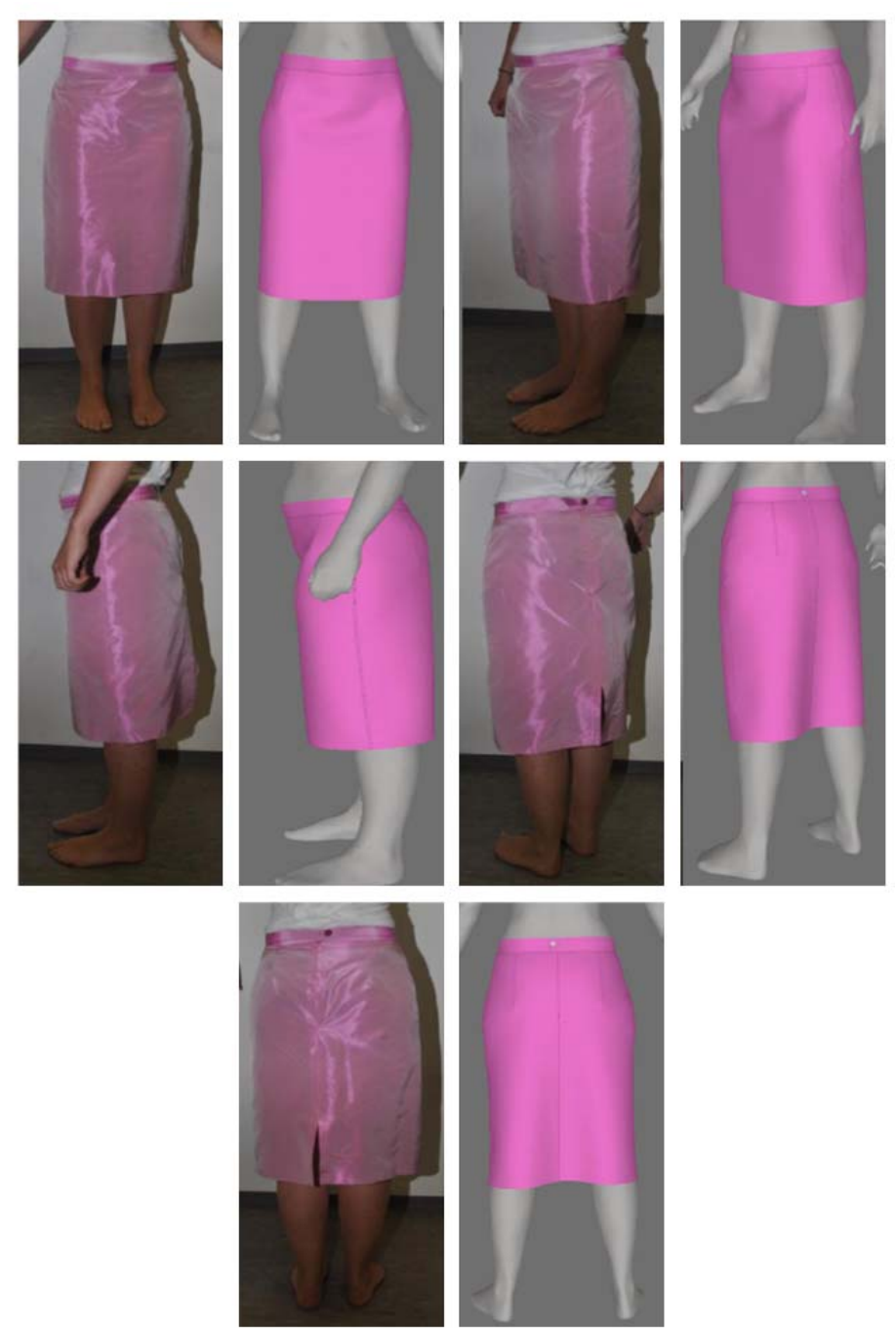

Fig.12: Results of virtual fitting on scanatar versus real fitting

body distance colour mapping: blue minimum $0 \mathrm{~mm}$; red maximum $30 \mathrm{~mm}$; material $\mathrm{M}_{3}$

\section{Example 3 - construction faults and results}

The figures below show 2 faults detected by 3D and caused by construction.

The skirt shown in figure 15 has a poor fit because of the wrong choice of ease for the waist measurement- usually the measurement was $1 / 2$ hip $+1 \mathrm{~cm}$ - in this case it was $1 / 2$ hip $+4 \mathrm{~cm}$ - in total 8 $\mathrm{cm}$ plus to waist. Therefore the skirt runs down to the hip line- already visible in the simulation.
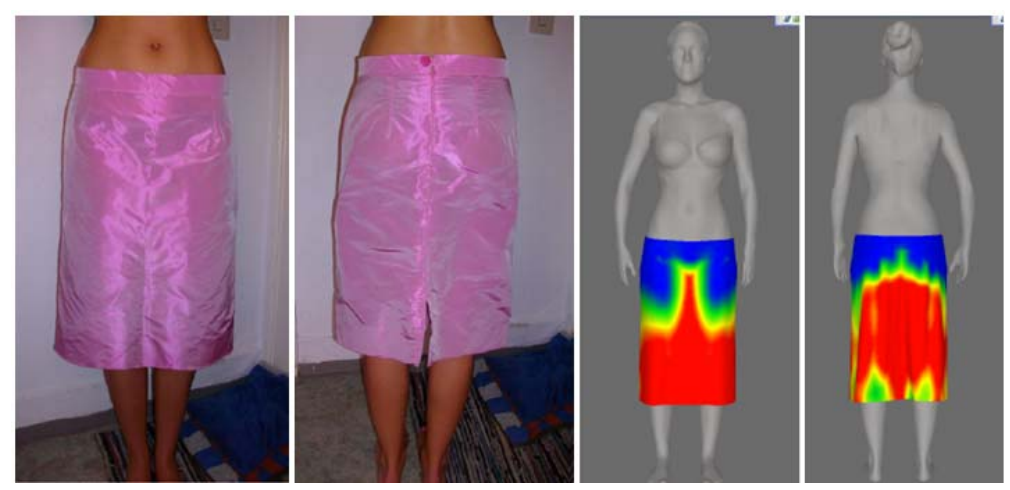

Fig.13: Wrong ease for waist- virtual versus reality; material $M_{3}$ 
The next example shows the wrong distribution for the individual pattern with regard to the dart content of the front and the back panel- therefore the "belly" becomes visible.

Therefore the distribution given in the construction systems should always be adapted to individual body measurements and shapes to achieve a well fitted individual skirt. Thus not only the measurements should be taken into account but also the additional information delivered from 3D body scan should be respected for construction.

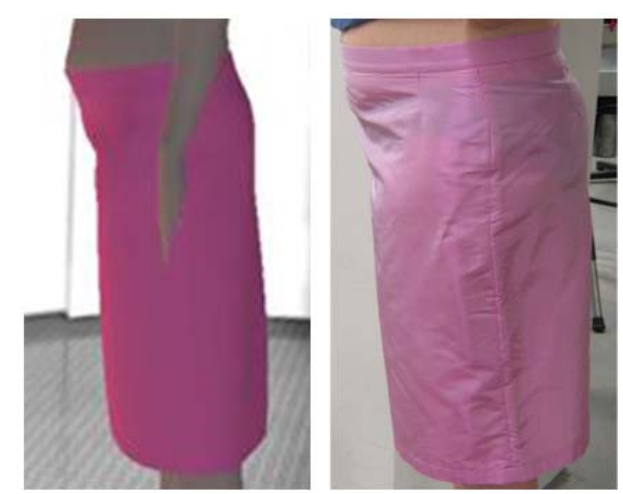

Fig.14: Wrong distribution of waist to hip ratio-virtual versus reality, material $M_{3}$

\section{Discussion and Conclusion}

The way from individual scanning of human subjects, generating scanatars and using them for virtual fit control in a 3D CAD tool was successfully tested in a first approach.

The simulation of different basic block constructions for skirts using latest SizeGERMANY measurements and SizeGERMANY avatars show only few variations by achieving good results in fit control. Most influence is caused by different characteristics of material and is part of future work.

Therefore also further investigations of the materials, the parameters and the practicability are already ongoing.

Selecting the appropriate individual measurements from the body scan and running through a common basic construction of skirt leads to good and feasible results of the virtual fit control on the generated scantars in comparison to the real sewn apparel.

If the body measurements and shapes differ largely from the normal distribution and ratios between the measurements are too high or to low, typical pattern construction problems occur and require expertise in adapting the blocks.

Transferring the presented virtual product development process design into the ready-made clothing segment a virtual try-on at the virtual mirror breaks new grounds in future:

In the Virtual Mirror (Fig. 15), developed by Human Solutions, customers can try on the new collection in the shop right away, without having to enter the changing room. The body scanner first creates a 3D model of the customer. The digital customer and the CAD data of the garments are then brought together in the Virtual Mirror. Now the (real) customer can configure and try on different styles and garments to his hearts' content, ultimately choosing his favorite. And this virtual, life-size try-on naturally works on digital house models during product development. 


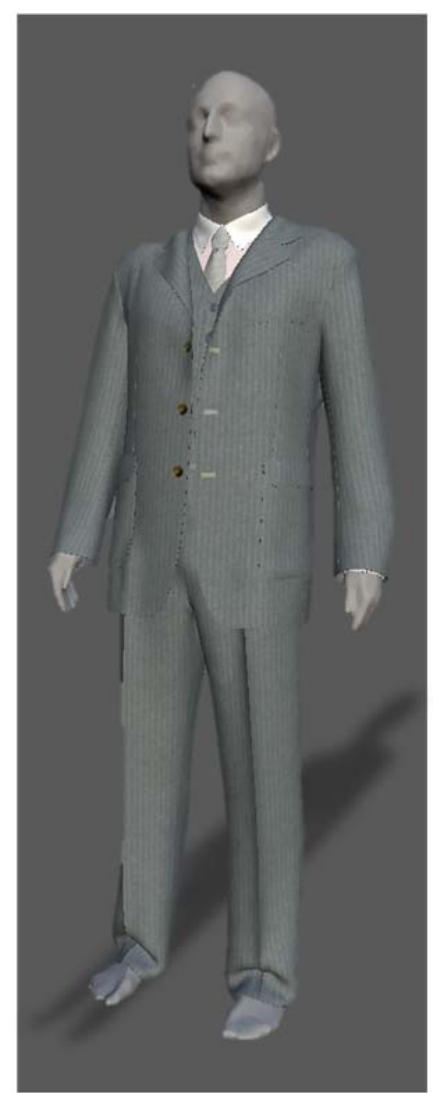

Fig.15: Virtual mirror: Scanatar with simulated suit

\section{References}

1. Ernst, M., (2011) "Technical Textile Product Development Using Innovative CAD Technology", Cape Peninsula University of Technology, Cape Town, South Africa, July 3, 2011

2. Ernst, M., (2011): "Sizing and Fit- 3D Body Scanning", Cape Peninsula University of Technology, Cape Town, South Africa, March 14, 2011

3. Ernst, M., (2010): "3D CAD Simulation Tools for Clothing Industry", Proceedings Assyst Fashion Forum, Munich, Germany

4. Ernst, M, (2009): "Powerful- 3D CAD in Application": Textile Network 4/09, pp. 20-21.

5. Ernst, M., (2008): "3D CAD- ways to Rapid Prototyping", fashion technics today, 05/08, pp. 12-13.

6. Hansen, G.; Trieb, R.: „AVANTI 3D. Entwicklung und Realisierung von Methoden und Verfahren zur schnellen Generierung statistisch korrekter dreidimensionaler Avatare auf Basis von 3D Body Scans." Abschlussbericht zum Forschungsvorhaben

7. Lim, H. S.: "Three Dimensional Virtual Try-on Technologies in the Achievement and Testing of Fit for Mass Customization." Dissertation to Graduate Faculty of North Carolina State University, Textile Technology and Management, Raleigh, 2009

8. Liu, Y.-L.; Thang, D.-L.; Yuen, M. M.-F.: "A survey on CAD methods in 3D garment design." Computer in Industry 61 (2010) p. 576-593

9. Rissiek, A.; Trieb, R.: "iSize - Implementation of international anthropometric survey results for worldwide sizing and fit optimization in the apparel industry." International Conference on 3D Body Scanning Technologies, Lugano, Switzerland, 19-20 October 2010

10. Rissiek, A.; Trieb, R.: "FIGURA - The individual fashion manikin for collection development and ensuring garment fit." White Paper, 2006

11. Seidl, A: "The real thing - The future of 3D modelling and pattern design." IACDE conference, Venice, 2010 